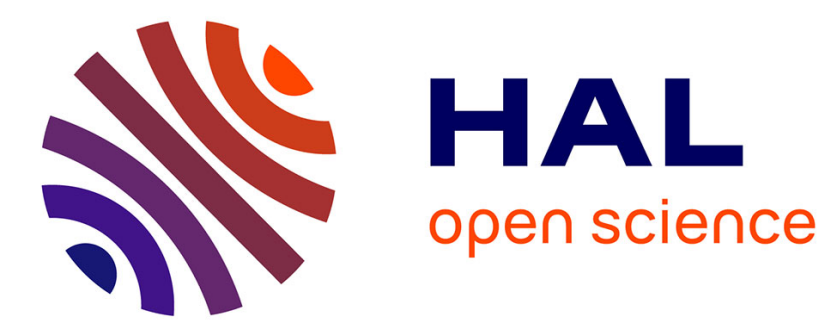

\title{
"Matreshka" model of multilayered current sheet
}

L.M. Zelenyi, H.V. Malova, V.Yu. Popov, Dominique C. Delcourt, N.Yu. Ganushkina, A.S. Sharma

\section{To cite this version:}

L.M. Zelenyi, H.V. Malova, V.Yu. Popov, Dominique C. Delcourt, N.Yu. Ganushkina, et al.. "Matreshka" model of multilayered current sheet. Geophysical Research Letters, 2006, 33 (5), pp.L05105. 10.1029/2005GL025117 . hal-00156510

\section{HAL Id: hal-00156510 https://hal.science/hal-00156510}

Submitted on 8 Feb 2016

HAL is a multi-disciplinary open access archive for the deposit and dissemination of scientific research documents, whether they are published or not. The documents may come from teaching and research institutions in France or abroad, or from public or private research centers.
L'archive ouverte pluridisciplinaire HAL, est destinée au dépôt et à la diffusion de documents scientifiques de niveau recherche, publiés ou non, émanant des établissements d'enseignement et de recherche français ou étrangers, des laboratoires publics ou privés. 


\title{
"Matreshka" model of multilayered current sheet
}

\author{
L. M. Zelenyi, ${ }^{1}$ H. V. Malova, ${ }^{1,2,3}$ V. Y. Popov, ${ }^{4}$ D. C. Delcourt, ${ }^{3}$ N. Y. Ganushkina, ${ }^{5}$ \\ and A. S. Sharma ${ }^{6}$ \\ Received 3 November 2005; revised 26 November 2005; accepted 11 January 2006; published 14 March 2006.
}

[1] Current sheets are one of the key elements of the Earth's magnetosphere, determining the magnetic energy storage and release. An analytical self-consistent model of multicomponent thin current sheets, where the plasma consists of ions of both solar wind and ionospheric origin, is presented. The influence of the electron population is taken into account assuming Boltzman-like quasi-equilibrium distribution in the electrostatic field, which can lead to a sharp peak in the electron current density in the center of the current sheet. We include the contribution of non-adiabatic $\mathrm{O}^{+}$ions in our model as one of the terms in the Grad-Shafranov-like system of equations describing the quasi-equilibrium configuration. The contribution of the oxygen ions to the total cross-tail current usually does not exceed $30 \%$ for realistic conditions, but current "wings" carried by $\mathrm{O}^{+}$ions produce significant broadening of the current profile. Citation: Zelenyi, L. M., H. V. Malova, V. Y. Popov, D. C. Delcourt, N. Y. Ganushkina, and A. S. Sharma (2006), "Matreshka" model of multilayered current sheet, Geophys. Res. Lett., 33, L05105, doi:10.1029/2005GL025117.

\section{Introduction}

[2] Recent in-situ measurements by CLUSTER spacecrafts [Sergeev et al., 2003; Runov et al., 2003; Asano et al., 2005] demonstrated that current sheets in the Earth's magnetotail can be as thin as 1-2 ion Larmor radii and have complicated internal structures with a hierarchy of spatial scales [Sergeev et al., 1993]. As a rule, the crosstail current profiles are substantially different from the well-known Harris model [Harris, 1962]. Although the plasma mantle has been considered as a primary source for populating the Earth's magnetotail [Pilipp and Morfill, 1978], the composition measurements of the magnetospheric plasma [Lennartsson and Shelley, 1986] have shown the important role of particles of ionospheric origin.

[3] The ion composition measurements in the lobes at distances $10 R_{E}>X>22 R_{E}$ have shown the presence of relatively low energy $(\sim 10 \mathrm{eV}-1 \mathrm{keV})$ heavy ions

\footnotetext{
${ }^{1}$ Space Research Institute, Russian Academy of Sciences, Moscow, Russia.

${ }^{2}$ Nuclear Physics Institute, Moscow State University, Moscow, Russia

${ }^{3}$ Centre d'etudes des Environnements Terrestres et Planetaires-Centre National de la Recherche Scientfique, Saint-Maur des Fausses, France.

${ }^{4}$ Physics Department, Moscow State University, Moscow, Russia.

${ }^{5}$ Finnish Meteorological Institute, Helsinki, Finland.

${ }^{6}$ Department of Astronomy, University of Maryland, College Park, Maryland, USA.
}

Copyright 2006 by the American Geophysical Union. 0094-8276/06/2005GL025117 streaming anti-sunward [Vaisberg et al., 1996; Delcourt et al., 1996; Sauvaud and Kovrazhkin, 2004]. It was demonstrated that $O^{+}$ions dominate the plasma sheet during substorms and their contribution to the current across magnetotail could be substantial. One should note here that generally the density of oxygen current might depend on the history of the system, e.g., if $\mathrm{O}^{+}$ions are ejected from the ionosphere without any substantial momentum in the main current direction, the momentum conservation will prevent a formation of strong oxygen currents. We are not considering here the mechanisms of magnetosphere population by ionospheric ions, but in our modelling of the magnetotail current sheet are taking into account its multicomponent composition including solar wind $\left(\mathrm{H}^{+}, \mathrm{He}^{++}\right)$and ionospheric $\left(\mathrm{O}^{+}, \mathrm{He}^{+}\right)$ ions. Until now the effects of such complicated compositions both on the structure of kinetic quasi-equilibria and dynamical processes are not well understood. Recently Kistler et al. [2005] estimated the upper limit for the contribution of oxygen ions to the total cross-tail current to be as much as $10 \%$. This is already a considerable effect and we show in this paper the important consequences of the presence of oxygen ions, viz. it dominates the cross-tail current outside the rather narrow tail midplane.

[4] We present here a quantitative analysis of the contributions of $\mathrm{O}^{+}$ions to the structure of Vlasov equilibria in the magnetotail plasma. In this first attempt to model the effect of ions other than $H^{+}$, we will neglect other relatively minor ions, such as $\mathrm{He}^{+}$and $\mathrm{He}^{++}$. Using the semi-analytical kinetic model of magnetotail Vlasov quasiequilibria developed earlier [Sitnov et al., 2000; Zelenyi et al., 2000, 2004a] we study the dependence of the shape of the cross-tail current profile on a number of parameters, viz. $B_{n} / B_{0}, n_{O+} / n_{H+}, T_{O+} / T_{H+}, T_{e \perp} / T_{i}, T_{e \|} / T_{e \perp}$. Here $B_{n}$ is the normal component of the magnetic field and $B_{0}$ is the total magnetic field, $n$ and $T$ are the density and temperature (parallel and perpendicular) of the plasma components, respectively.

\section{Multi-component Model of the Magnetotail Current Sheet}

[5] We develop in this paper a self-consistent equilibrium model of $1 D$ anisotropic current sheet with a small normal component of the magnetic field $B_{z}$, in the GSM system of reference. The tension of the curved magnetic field lines is balanced by the centrifugal force acting on ions crossing the sheet midplane, leading to a $1 \mathrm{D}$ equilibria with $\partial / \partial x=0$ (i.e., with no gradients along the tail axis). The plasma in the source region at the edges of the system move toward the central region (as shown in Figure 1) where they carry a cross-tail current ( $J_{y}$ in our geometry) consistent with the magnetic field reversal in the vicinity of the $z=0$ plane. The 


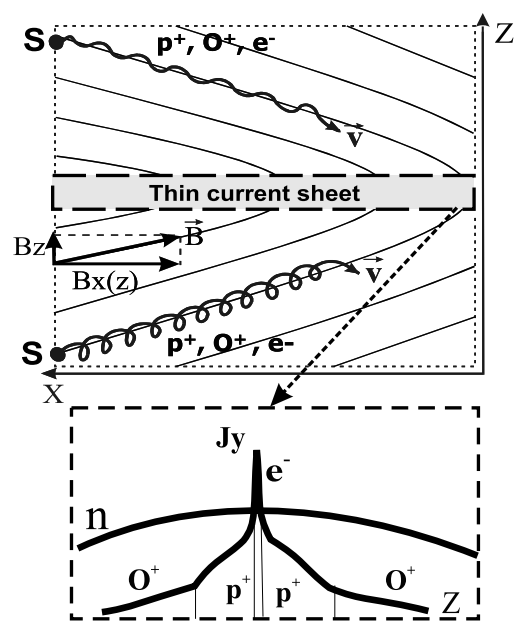

Figure 1. The general scheme of 1D model of thin current sheet. (top) Plasma particles cross CS moving from the edges of the system toward the center. (bottom) Four levels of current sheet embedding are shown.

electric field component $E_{y}$ can be neglected without loss of generality by selecting a suitable deHoffmann-Teller frame of reference. The dynamics of ions and electrons are different inside the thin current sheet (TCS), so one can use a hybrid approach to describe the behavior of the different particle species. The non-adiabatic hydrogen $H^{+}$ and oxygen $O^{+}$ions moving across the sheet with Speiser orbits will be described in the so called quasiadiabatic approximation [Sitnov et al., 2000; Zelenyi et al., 2000]. The electrons are considered as magnetized particles with finite inertia for motion across field lines and negligible inertia for field-aligned motion. The resulting charge separation generates an ambipolar electric field $E_{z}$ supporting the quasineutrality of the plasma. The problem is then reduced to the solution of a Grad-Shafranov type equation, where currents from all particles species are selfconsistently calculated from the equations of particles motion in the electric and magnetic fields generated by these currents.

[6] When ions cross the current sheet (CS) region, their motion is characterized by three integrals of motion: the total energy $W_{0}=m\left(v_{x}^{2}+v_{y}^{2}+v_{z}^{2}\right) / 2+e \phi$, the canonical momentum $P_{y}=m v_{y}-(e / c) A_{y}(z)$ and the approximate integral of motion $I_{z}=\frac{1}{2 \pi} \oint m v_{z} d z$ (here $\vec{v}$ is the particle velocity, $A_{y}(z)$ is the vector-potential, and $\phi$ is the electrostatic potential). $I_{z}$, the so called Speiser CS invariant, is approximately conserved in a TCS where particle oscillations along $z$ and $x$ coordinates are decoupled because of the widely separated oscillation frequencies: $\omega_{x} / \omega_{z} \ll 1$ [Buchner and Zelenyi, 1989; Chen, 1992]. In the quasiadiabatic approximation, the invariant $I_{z} \approx$ const is used to render the equations of motion integrable and to obtain the ion distribution at any position using Liouville theorem. As mentioned above, the value of $B_{n}$, the normal component of the magnetic field is a small parameter with a specified value, and the only assumption is that it is strong enough to keep the electrons magnetized. In this paper we set $B_{z}=0.1 B_{0}$, and the electron motion along the field lines is assumed to be fast enough to support a quasi-equilibrium
Boltzmann distribution in the presence of an electrostatic potential and mirror forces.

\section{Basic Equations of the Model}

[7] The CS model in the presence of $O^{+}$is derived following of our earlier theoretical model where only two plasma components, protons and electrons, were taken into account [Zelenyi et al., 2004a, 2004b]. The Maxwell equation in the 1D case takes the simple form:

$$
\frac{d B(x)}{d z}=\frac{4 \pi}{c}\left(j_{H}+j_{O}+j_{e}\right)
$$

[8] The magnetic field $B_{x}$ in the TCS arises from the currents carried by $\mathrm{H}^{+}, \mathrm{O}^{+}$and electrons $e^{-}$. The boundary condition is $B_{x}(\infty)=B_{0}$ and the current densities $j_{H}$ and $j_{O}$ are

$$
j_{H, O}(z)=e \int_{0}^{\infty} d v_{x} \int_{0}^{\infty} d v_{z} \int_{0}^{\infty} v_{y} f_{H, O}(\vec{v}, z) d v_{y}
$$

with the distribution function $f_{H, O}$ to be a shifted Maxwellian function at the position of plasma source, i.e., at the boundary of the system $z= \pm L$ (Figure 1): $f_{H, O} \sim \exp \left\{-\left[\left(v_{\|}-v_{D_{H . O}}\right)^{2}+v_{\perp}^{2}\right] / v_{T_{H, O}}\right\}$ (where $v_{T_{H, O}}$ are the thermal velocities, and $v_{D_{H, O}}$ are the drift velocities of $\mathrm{H}^{+}$or $\mathrm{O}^{+}$ions). The electron currents are taken into account, with the pressure anisotropy $\left(p_{\|} \neq p_{\perp}\right)$ which corresponds to the curvature drift of electrons in the reversed magnetic field:

$$
\begin{aligned}
j_{e \perp}= & -e n_{e} c \frac{[\vec{E} \times \vec{h}]}{B}+\frac{c}{B}\left[\vec{h} \times \vec{\nabla}_{\left.\perp p_{\perp e}\right]}\right. \\
& +\frac{c}{B}\left(p_{I I e}-p_{\perp e}\right)[\vec{h} \times(\vec{h} \vec{\nabla}) \vec{h}], \vec{h}=\frac{\vec{B}}{B}
\end{aligned}
$$

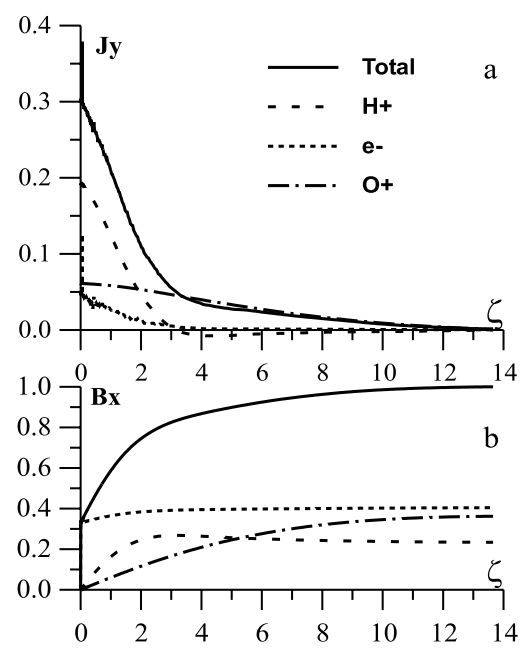

Figure 2. Total and partial current densities $J_{y}=j_{y} /\left(e n_{0} v_{D_{H}}\right)$ (a) and corresponding magnetic fields (b) in TCS as a function of dimensionless coordinate $\zeta \sim Z / \rho_{i}$. 


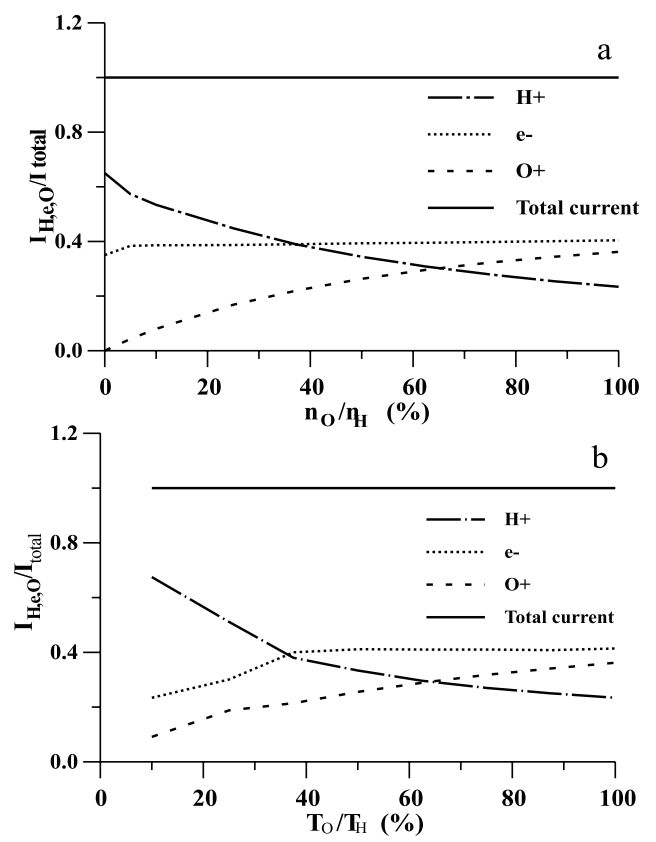

Figure 3. Total current as a function of plasma parameters (a) of the relative content of $\mathrm{O}^{+}$ions in three component plasma and (b) of the oxygen to hydrogen temperature ratio.

Here $B=|B( \pm \infty)|, \vec{E}$ is the ambipolar electric field $E_{z}$. The detailed description of a semi-hydrodynamic approach for obtaining the electron current $j_{e}$, the electric field $E_{z}(z)$ and its influence on electrons and protons is given in our earlier paper [Zelenyi et al., 2004a]. Finally we solve the full system of equations for $B_{x}, j_{y}, \phi$ and $n$ (plasma density) using the integrals of motion $W_{0}, P_{y}$ and $I_{z}$.

\section{Results and Conclusions}

[9] The self-consistent system of equations for the magnetic and electric fields, total and partial current densities as well as electrostatic potential and plasma densities were solved numerically using a two-step iteration procedure for ions and electrons [Zelenyi et al., 2004a, 2004b]. The results of this model are shown in Figure 2, where the profiles of the current densities and the magnetic fields of the different plasma constituents and the total profiles are shown. The parameters of the system were chosen to be close to the observed values, e.g., the oxygen to hydrogen temperature ratio outside the sheet are $T_{0} / T_{H}=1,0.5,0.25,0.1$, and 0.0 . The initial density of oxygen is varied as $(0,0.1,0.25,0.5$, 1.0) $n_{H}$ outside the sheet. In Figure 2 one of these cases where $T_{O^{+}} / T_{H^{+}}=1$ and $n_{O^{+}} / n_{H^{+}}=1$ are shown for the reference. The role of electrons is most substantial in the center of CS, where they dominate and support a very narrow and peaked current embedded in the proton current, which in turn is embedded in the oxygen current. The currents of $\mathrm{O}^{+}$are comparable with that of $\mathrm{H}^{+}$, but the halfthickness of the oxygen current density $\left(\sim 6 \rho_{L}\right)$ is three times larger than that of the protons $\left(\sim 2 \rho_{L}\right)$. It is clear from Figure 2 that both the ion populations contribute approximately equally to the total cross-tail current, although the total CS thickness is determined generally by the oxygen ion current. Also the net contribution of $\mathrm{O}^{+}$-current density is seen to be about $30 \%$ of the total current density.

[10] As the role of $O^{+}$in Figure 2 could be somewhat exaggerated, the parameters were varied over a wider range and the results are shown in Figure 3. The observations [Vaisberg et al., 1996; Delcourt et al., 1996; Sauvaud and Kovrazhkin, 2004] show that the relative fraction of oxygen in the magnetotail may vary widely during different phases of magnetospheric activity (but very rarely exceed the density of hydrogen ions) and accordingly we have investigated the dependence of $I_{O} / I_{\text {total }}$ (where $I_{O}$ and $I_{\text {total }}$ are the $\mathrm{O}^{+}$and total currents, respectively) as a function of the ratio of densities $n_{O}$ to $n_{H}$ (Figure $3 \mathrm{a}$ ) and of the temperature ratio $T_{O} / T_{H}$ (Figure $3 \mathrm{~b}$ ). An increase of oxygen ion content in the magnetotail plasma from $25 \%$ to $100 \%$ leads to an enhancement of its the relative contribution to cross-tail current from $18 \%$ to $\sim 30 \%$. Similar results are achieved if one decreases $O^{+}$temperature in comparison with that of $H^{+}$. Here we also see that the oxygen current contribution, although being sensitive to $O^{+}$temperature, still remain in the $(20-30) \%$ range.

[11] The contributions of oxygen ions to the magnetotail current, shown in Figure 3 can be compared with the estimates of the oxygen currents in the magnetotail from the spacecraft observations. Estimates using Cluster measurements [Kistler et al., 2005] yield the relative density of oxygen ions to be of the order of $15 \%$. One should mention that our estimate of $30 \%$ is the upper limit, and it depends strongly on the oxygen flux at the source and on the oxygen temperature. In general our results provide estimates of the same order as the observations. Our principal conclusion concerns the role of oxygen in the formation of the local structure of thin current sheets in the Earth's magnetotail. The local current of $O^{+}$ions leads to an effective thickening of the total current profile and is dominant in the outer regions of the sheet.

[12] A combination of the contributions of the oxygen ions, presented in this paper, and our earlier models of TCS [Zelenyi et al., 2004a, 2004b], yields a multilayer structure of the current sheet resembling a "matreshka", a popular Russian nesting doll. A very thin electron layer $L \sim \rho_{i}\left(B_{n} / B\right)^{4 / 3} \sim 0.05-0.1 \rho_{i}$ is embedded in a thin proton sheet with $L \sim \rho_{i}$, which in turn is embedded in a thick oxygen sheet $\left(L \sim 7-10 \rho_{i}\right)$. Further, the CS as whole is embedded inside the thicker plasma sheet [Zelenyi et al., 2004a, 2004b], i.e., generally there are four levels of embedding (Figure 1).

[13] Acknowledgment. This work was supported by the RFBR grants 05-02-17003, 04-02-17371, 05-05-64993, RFBR GFEN 04-02-39021, INTAS grant 03-51-3738, grant of Scientific schools HIII-1739.2003.2 and NASA grant NNG 04GE37G.

\section{References}

Asano, Y., R. Nakamura, W. Baumjohann, A. Runov, Z. Voros, M. Volwerk, T. L. Zhang, A. Balogh, B. Klecker, and H. Reme (2005), How typical are atypical current sheets?, Geophys. Res. Lett., 32, L03108, doi:10.1029/ 2004GL021834.

Buchner, J., and L. M. Zelenyi (1989), Regular and chaotic charged particle motion in magnetotaillike field reversals: 1. Basic theory of trapped motion, J. Geophys. Res., 94, 11,821.

Chen, J. (1992), Nonlinear dynamics of charged particles in the magnetotail, J. Geophys. Res., 97, 15,011.

Delcourt, D. C., J.-A. Sauvaud, O. L. Vaisberg, L. A. Avanov, J. L. Burch, and J. H. White (1996), Signatures of impulsive convection in the magnetospheric lobes, Geophys. Res. Lett., 23, 129. 
Harris, E. G. (1962), On a plasma sheath separating regions of oppositely directed magnetic fields, Nuovo Cimento, 23, 115.

Kistler, M., et al. (2005), Contribution of nonadiabatic ions to the cross-tail current in an $\mathrm{O}^{+}$dominated thin current sheet, J. Geophys. Res., 110, A06213, doi:10.1029/2004JA010653.

Lennartsson, W., and E. G. Shelley (1986), Survey of 0.1- to 16-keV/e plasma sheet ion composition, J. Geophys. Res., 91, 3061.

Pilipp, W. G., and G. Morfill (1978), The formation of the plasma sheet resulting from plasma mantle dynamics, J. Geophys. Res., 83, 5670.

Runov, A., et al. (2003), Current sheet structure near magnetic X-line observed by Cluster, Geophys. Res. Lett., 30(11), 1579, doi:10.1029/ 2002GL016730.

Sauvaud, J.-A., and R. A. Kovrazhkin (2004), Two types of energydispersed ion structures at the plasma sheet boundary, J. Geophys. Res., 109, A12213, doi:10.1029/2003JA010333.

Sergeev, V. A., D. G. Mitchell, C. T. Russell, and D. J. Williams (1993), Structure of the tail plasma/current sheet at $11 \mathrm{Re}$ and its changes in the course of a substorm, J. Geophys. Res., 98, 17,345.

Sergeev, V., et al. (2003), Current sheet flapping motion and structure observed by Cluster, Geophys. Res. Lett., 30(6), 1327, doi:10.1029/ 2002 GL016500.

Sitnov, M. I., L. M. Zelenyi, H. V. Malova, and A. S. Sharma (2000), Thin current sheet embedded within a thicker plasma sheet: Self-consistent kinetic theory, J. Geophys. Res., 105, 13,029.

Vaisberg, O. L., L. A. Avanov, J. L. Burch, and J. H. Waite Jr. (1996), Measurements of plasma in the magnetospheric tail lobes, Adv. Space Res., 8, 63 .
Zelenyi, L., M. I. Sitnov, H. V. Malova, and A. S. Sharma (2000), Thin and superthin ion current sheets, quasiadiabatic and nonadiabatic models, Nonlinear Processes Geophys, 7, 127.

Zelenyi, L. M., H. V. Malova, V. Y. Popov, D. C. Delcourt, and A. S. Sharma (2004a), Nonlinear equilibrium structure of thin currents sheets: Influence of electron pressure anisotropy, Nonlinear Processes Geophys, 11,1 .

Zelenyi, L. M., H. V. Malova, V. Y. Popov, D. C. Delcourt, and A. S. Sharma (2004b), Role of electrostatic effects in thin current sheets, Multiscale Processes in the Earth's Magnetosphere: From Interball to Cluster, NATO Sci. Ser., vol. 178, edited by J.-A. Sauvaud and Z. Nemecek, pp. 275-288, Kluwer Academic Publishers.

D. Delcourt, Centre d'etudes des Environnements Terrestres et Planetaires-CNRS, F-94107 Saint-Maur des Fausses, France. (dominique. delcourt@cetp.ipsl.fr)

N. Ganushkina, Finnish Meteorological Institute, FIN-00101 Helsinki, Finland. (natalia.ganushkina@fmi.fi)

H. Malova, Nuclear Physics Institute, Moscow State University, 119992, Moscow, Russia. (mlv@dec1.npi.msu.su)

V. Popov, Physics Department, Moscow State University, 119992, Moscow, Russia. (masterlu@mail.ru)

A. S. Sharma, Department of Astronomy, University of Maryland, College Park, MD 20742, USA. (ssh@astro.umd.edu)

L. Zelenyi, Space Research Institute, RAS, Profsoyusnaya street 84/32, 117810, Moscow, Russia. (lzelenyi@iki.rssi.ru) 